\title{
Original
}

\section{Three-dimensional craniofacial morphology in unilateral cleft lip and palate}

\author{
Eyas Abuhijleh1), Halise Aydemir2), and Ufuk Toygar-Memikoğlu3) \\ 1)Tawam Hospital in Affiliation with Johns Hopkins Medicine International Dental Center, \\ Abu Dhabi, United Arab Emirates \\ 2)Turgut Özal University Oral and Dental Health Care Center, Ankara, Turkey \\ ${ }^{3)}$ Department of Orthodontics, Faculty of Dentistry, Ankara University, Ankara, Turkey
}

(Received November 11, 2013; Accepted May 6, 2014)

\begin{abstract}
Craniofacial morphology and asymmetry were compared before, during, and after puberty within and between patients with unilateral complete cleft lip and palate (UCCLP) and a non-cleft group. In the UCCLP group, the posterior cranial base and total cranial base were significantly shorter at all skeletal periods, the maxilla was significantly retruded and posteriorly rotated, and the mandible was significantly smaller and inferoposteriorly rotated. The angle between the nasal and mandibular plane and lower anterior facial height were significantly higher, and upper posterior facial height and total posterior height were significantly lower, in the UCCLP group. Except for an increase in the nasal cavity, no significant differences were detected in facial width. For all measurements, asymmetry on the horizontal plane was more significant than that on the vertical plane. Asymmetries in the UCCLP group were mostly detected during puberty. The UCCLP group had no distinctive mandibular asymmetry, as compared with the Class I group. In UCCLP patients, the cranial base, maxilla, and mandible were affected on the sagittal plane during all growth periods. However, horizontal asymmetries were mostly detected before and during puberty. Vertical asymmetries were less severe, and there was no distinctive mandibular asymmetry as
\end{abstract}

Correspondence to Dr. Halise Aydemir, Turgut Özal University Oral and Dental Health Care Center, Alparslan Türkeş Caddesi No: 57 Emek, Ankara, Turkey

E-mail: hsbolatoglu@yahoo.com

doi.org/10.2334/josnusd.56.165

DN/JST.JSTAGE/josnusd/56.165 compared with the Class I group.

(J Oral Sci 56, 165-172, 2014)

Keywords: craniofacial morphology; cleft lip palate; asymmetry.

\section{Introduction}

Cleft lip and palate (CLP), one of the most common congenital craniofacial anomalies, has a multifactorial etiology. CLP affects the functions of the stomatognathic system and disrupts dentofacial esthetics. Knowledge of craniofacial growth and morphology in CLP patients is crucial in early treatment planning.

Operated patients with CLP generally have craniofacial deformities, especially due to defects in the growth of the nasomaxillary complex, depending on cleft type and surgical procedure (1). Thus, to provide appropriate treatment during all growth periods it is important to understand the craniofacial growth and morphologic characteristics of such patients.

Typical manifestations of CLP include a more retrognathic and posteriorly inclined maxilla (with decreased length and midfacial deficiency related to the retruded maxilla), greater flattening of the cranial base, a larger mandibular plane and gonial angle, larger anterior facial height, and decreased posterior facial height (2). On the horizontal plane, maxillary bone defects and distortions are common (3-5), and patients show distinct facial asymmetry, even after corrective surgery (6). However, the extent of mandibular asymmetry is disputed (7).

Previous studies have mostly evaluated sagittal and vertical relationships in craniofacial morphology, espe- 
cially in the nasomaxillary complex $(2,8,9)$. Computed tomography was used to evaluate facial asymmetry and sagittal and vertical facial morphology; however, these studies were uncontrolled and restricted to a small number of CLP patients $(7,10,11)$. The differences in the three-dimensional (sagittal, horizontal and vertical planes) growth characteristics of CLP patients and Class I individuals are a matter of controversy.

We used lateral and posteroanterior (PA) cephalograms to examine the craniofacial morphology of patients with unilateral complete cleft lip and palate (UCCLP) in the sagittal, vertical, and horizontal planes. In addition, we evaluated asymmetries during three growth periods and compared those findings to those obtained from a control group of patients without cleft. To our knowledge, this is the first study with an age-matched control group to assess CLP craniofacial morphology before, during,

Table 1 Age and number distributions of patients, according to group and growth period

\begin{tabular}{lccc}
\hline Group & Growth period & No. & Age $(\mathrm{X} \pm$ Sx $)$ \\
\hline $\operatorname{UCCLP}(n=59)$ & T1 & 24 & $9.65 \pm 1.82$ \\
& T2 & 17 & $13.51 \pm 1.39$ \\
Control $(n=77)$ & T3 & 18 & $17.08 \pm 2.12$ \\
& T1 & 26 & $11.27 \pm 1.29$ \\
& T2 & 26 & $13.28 \pm 1.24$ \\
& T3 & 25 & $16.15 \pm 0.57$ \\
\hline
\end{tabular}

X: Mean, Sx: Standard error of the mean

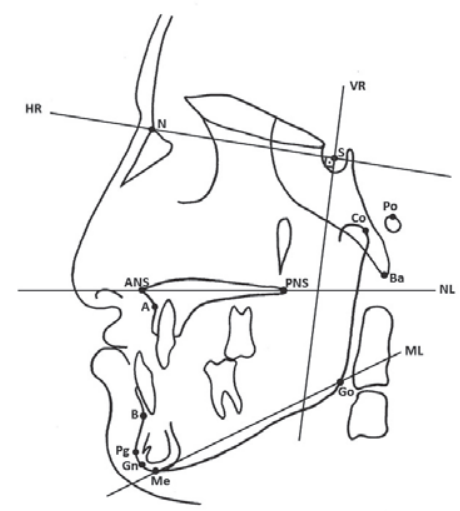

Fig. 1 Lateral cephalometric points and planes. S-N (mm), anterior cranial base; S-Ba $(\mathrm{mm})$, posterior cranial base; N-Ba $(\mathrm{mm})$, total cranial base; N.S.Ba $\left(^{\circ}\right)$, cranial base angle; Co-A $(\mathrm{mm})$, effective maxillary length; Go-Gn ( $\mathrm{mm})$, mandibular corpus; Co-Gn (mm), mandibular effective length; Co-Go (mm), mandibular ramus; N-ANS (mm), upper anterior facial height; ANS-Me (mm), lower anterior facial height; N-Me (mm), anterior facial height; S-PNS (mm), upper posterior facial height; S-Go $(\mathrm{mm})$, posterior facial height. and after puberty on the vertical, sagittal, and horizontal planes.

\section{Materials and Methods}

This cross-sectional study enrolled 59 Turkish patients (age, 6-22 years) with UCCLP and no other congenital anomaly or syndrome. CLP patients were selected from among patients receiving orthodontic therapy at Ankara University, Faculty of Dentistry, Department of Orthodontics. None of the UCCLP patients had received previous orthodontic or orthopedic therapy. All had undergone cleft surgery at the same center under the same protocol, namely, the lip was closed at age 3 months using the modified Millard rotation-advancement technique, and the palate was closed at age 9-12 months using a two-flap pushback palatoplasty. The operations were monitored by orthodontists (a research assistant and a professor/associate professor) and were performed by maxillofacial surgeons (research assistants and a professor/associate professor) specialized in CLP treatment. None of the UCCLP patients had a history of alveolar bone graft or pharyngeal flap surgery.

The control group comprised 77 Turkish children and adults (age, 8-20 years) classified as skeletal and dental Class I. Individuals who had received orthodontic therapy and those with any dentofacial anomalies were excluded from the control group. Radiographs from both groups were selected from the university orthodontic department archive. The Ethical Committee of the Ankara University

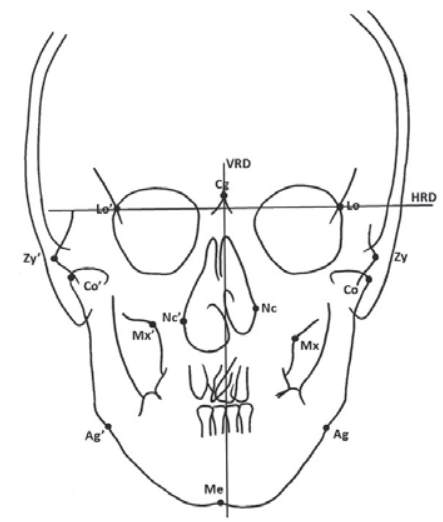

Fig. 2 PA cephalometric points and planes. $\mathrm{Nc}-\mathrm{Nc}$ ' $(\mathrm{mm})$, nasal width; Zy-Zy' (mm), zygomatic width; Mx-Mx' (mm), maxillary width; Co-Co' (mm), condylar width; Ag-Ag' (mm), antegonial width; $\mathrm{Ag}$ ( $\mathrm{Ag}$ ')-Me (mm), distance between points $\mathrm{Ag}\left(\mathrm{Ag}^{\prime}\right)$ and $\mathrm{Me}$; $\mathrm{Co}-\mathrm{Ag}\left(\mathrm{Co}^{\prime}-\mathrm{Ag}^{\prime}\right)(\mathrm{mm})$, distance between points $\mathrm{Co}$ and $\mathrm{Ag}$ (Co' and $\left.\mathrm{Ag}^{\prime}\right)$; $\mathrm{Co}$ (Co')-Me (mm), distance between points $\mathrm{Co}$ (Co') and $\mathrm{Me}$; Co.Ag.Me (Co'.Ag'.Me) (mm), angle between planes $\mathrm{CoAg}$ (Co'Ag') and $\mathrm{MeAg}(\mathrm{MeAg}$ ') measured at point $\mathrm{Ag}$. 
Faculty of Dentistry approved the study (approval, 2004).

Lateral cephalometric, PA cephalometric, and handwrist radiographs were used in the study. The hand-wrist radiographs were grouped according to growth period, as defined in the Greulich and Pyle atlas (T1: prepubertal,

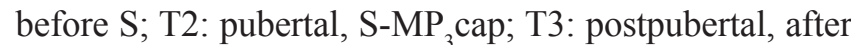
$\mathrm{MP}_{3}$ cap). The age and number distributions of the patients, according to growth period, are shown in Table 1.

\section{Cephalometric analysis}

All cephalometric radiographs were taken in centric occlusion, after swallowing, by the same technician using the same machine. Tracings were made in a blinded manner by one of the authors (E. A.), and cephalometric reference points were determined by using acetate paper.
Measurements were made with the help of the PORDIOS software program. Thirteen reference points, four planes, and 28 angular and linear measurements were used in the analysis of lateral cephalometric radiographs (Fig. 1). Fourteen reference points, two planes, and 33 angular and linear parameters were measured in PA cephalometric radiographs (Fig. 2). Asymmetry values were calculated as the vertical distances from the reference points to the vertical or horizontal planes.

\section{Reliability}

All cephalometric landmarks on radiographs were digitized twice by the same investigator, to eliminate measurement errors. All digitizing points and measurements were repeated on 80 randomly selected PA and

Table 2 Means and standard errors of the mean for lateral cephalometric radiograph variables at each growth period in the UCCLP and control groups

\begin{tabular}{|c|c|c|c|c|c|c|}
\hline & \multicolumn{6}{|c|}{ Groups } \\
\hline & \multicolumn{3}{|c|}{ UCCLP } & \multicolumn{3}{|c|}{ Control } \\
\hline & $\mathrm{T} 1(n=24)$ & $\mathrm{T} 2(n=17)$ & $\mathrm{T} 3(n=18)$ & $\mathrm{T} 1(n=26)$ & $\mathrm{T} 2(n=26)$ & $\mathrm{T} 3(n=25)$ \\
\hline & $\mathrm{X} \pm \mathrm{Sx}$ & $\mathrm{X} \pm \mathrm{Sx}$ & $\mathrm{X} \pm \mathrm{Sx}$ & $\mathrm{X} \pm \mathrm{Sx}$ & $\mathrm{X} \pm \mathrm{Sx}$ & $\mathrm{X} \pm \mathrm{Sx}$ \\
\hline \multicolumn{7}{|c|}{ Cranial measurements } \\
\hline $\mathrm{N}-\mathrm{S}$ & $67.80 \pm 0.66$ & $70.25 \pm 0.71$ & $72.68 \pm 0.92$ & $68.86 \pm 0.6$ & $70.69 \pm 0.72$ & $73.00 \pm 0.54$ \\
\hline S-Ba & $42.72 \pm 0.65$ & $45.89 \pm 0.82$ & $44.84 \pm 1.02$ & $44.38 \pm 0.69$ & $46.38 \pm 0.66$ & $47.02 \pm 0.86$ \\
\hline $\mathrm{N}-\mathrm{Ba}$ & $100.80 \pm 1.01$ & $105.84 \pm 1.17$ & $107.87 \pm 1.66$ & $103.49 \pm 0.97$ & $107.20 \pm 0.88$ & $109.84 \pm 0.93$ \\
\hline NSBa & $130.52 \pm 1.23$ & $130.42 \pm 1.42$ & $131.98 \pm 1.32$ & $131.01 \pm 0.83$ & $131.70 \pm 0.91$ & $131.60 \pm 1.18$ \\
\hline \multicolumn{7}{|c|}{ Maxillary measurements } \\
\hline Co-A & $80.61 \pm 1.03$ & $83.46 \pm 1.22$ & $84.29 \pm 1.61$ & $85.09 \pm 0.93$ & $88.02 \pm 1.02$ & $91.00 \pm 0.91$ \\
\hline A-VR & $56.31 \pm 1.33$ & $55.84 \pm 1.28$ & $56.72 \pm 1.43$ & $59.74 \pm 0.81$ & $60.87 \pm 1.19$ & $64.52 \pm 1.16$ \\
\hline A-HR & $54.11 \pm 0.80$ & $58.63 \pm 0.93$ & $60.74 \pm 1.50$ & $58.41 \pm 0.58$ & $60.08 \pm 0.73$ & $61.11 \pm 0.63$ \\
\hline SNA & $78.15 \pm 1.09$ & $76.25 \pm 1.14$ & $75.38 \pm 1.18$ & $81.12 \pm 0.58$ & $80.75 \pm 0.74$ & $82.15 \pm 0.75$ \\
\hline SN.NL & $12.13 \pm 0.89$ & $10.68 \pm 0.77$ & $12.69 \pm 1.13$ & $9.22 \pm 0.52$ & $9.00 \pm 0.78$ & $9.54 \pm 0.60$ \\
\hline \multicolumn{7}{|c|}{ Mandibular measurements } \\
\hline Go-Gn & $66.03 \pm 1.01$ & $71.02 \pm 1.59$ & $76.29 \pm 1.36$ & $71.48 \pm 0.76$ & $75.29 \pm 1.14$ & $80.77 \pm 1.13$ \\
\hline Co-Gn & $106.32 \pm 1.13$ & $115.0 \pm 51.75$ & $121.51 \pm 1.90$ & $111.57 \pm 0.83$ & $118.82 \pm 1.32$ & $123.23 \pm 1.13$ \\
\hline B-VR & $42.63 \pm 1.43$ & $45.24 \pm 2.69$ & $47.83 \pm 1.60$ & $48.33 \pm 1.14$ & $50.98 \pm 1.63$ & $55.14 \pm 1.36$ \\
\hline PG-VR & $40.84 \pm 1.58$ & $44.29 \pm 3.02$ & $47.37 \pm 1.88$ & $47.03 \pm 1.26$ & $50.21 \pm 1.82$ & $55.19 \pm 1.49$ \\
\hline Co-Go & $51.97 \pm 0.84$ & $57.10 \pm 1.39$ & $59.66 \pm 1.07$ & $55.60 \pm 0.63$ & $60.06 \pm 0.88$ & $63.03 \pm 0.71$ \\
\hline Go-HR & $67.15 \pm 1.03$ & $74.03 \pm 1.93$ & $76.19 \pm 1.41$ & $70.90 \pm 0.98$ & $76.68 \pm 1.13$ & $80.34 \pm 1.07$ \\
\hline Co-HR & $15.30 \pm 0.61$ & $17.18 \pm 0.91$ & $16.77 \pm 0.80$ & $15.44 \pm 0.61$ & $16.74 \pm 0.70$ & $17.46 \pm 0.69$ \\
\hline B-HR & $92.69 \pm 1.25$ & $99.02 \pm 1.90$ & $103.54 \pm 1.8$ & $95.43 \pm 0.92$ & $100.05 \pm 1.25$ & $101.78 \pm 0.95$ \\
\hline Pg-HR & $103.74 \pm 1.25$ & $112.08 \pm 1.93$ & $117.65 \pm 2.0$ & $106.89 \pm 0.98$ & $112.94 \pm 1.43$ & $114.78 \pm 1.08$ \\
\hline SNB & $74.85 \pm 0.74$ & $75.94 \pm 1.57$ & $76.54 \pm 0.7$ & $77.91 \pm 0.59$ & $78.88 \pm 0.75$ & $80.04 \pm 0.62$ \\
\hline SN.ML & $41.33 \pm 1.08$ & $39.87 \pm 1.62$ & $40.46 \pm 1.1$ & $36.86 \pm 0.90$ & $35.76 \pm 0.96$ & $33.01 \pm 0.67$ \\
\hline \multicolumn{7}{|c|}{ Maxillomandibular measurements } \\
\hline ANB & $3.30 \pm 0.83$ & $0.31 \pm 1.09$ & $-1.17 \pm 1.21$ & $3.21 \pm 0.25$ & $1.87 \pm 0.41$ & $2.11 \pm 0.42$ \\
\hline NL.ML & $26.46 \pm 0.98$ & $26.58 \pm 1.55$ & $25.05 \pm 1.24$ & $25.33 \pm 0.70$ & $24.08 \pm 1.02$ & $20.52 \pm 0.65$ \\
\hline CoGo.GoMe & $131.28 \pm 1.08$ & $130.92 \pm 1.88$ & $129.35 \pm 1.30$ & $124.81 \pm 0.81$ & $125.27 \pm 0.89$ & $120.54 \pm 0.71$ \\
\hline \multicolumn{7}{|c|}{ Facial height measurements } \\
\hline N-ANS & $50.29 \pm 0.64$ & $53.06 \pm 0.98$ & $55.84 \pm 1.30$ & $52.63 \pm 0.57$ & $55.07 \pm 0.64$ & $56.31 \pm 0.65$ \\
\hline ANS-Me & $65.89 \pm 1.14$ & $70.44 \pm 1.40$ & $72.98 \pm 1.34$ & $64.65 \pm 0.77$ & $68.52 \pm 1.02$ & $70.23 \pm 0.77$ \\
\hline $\mathrm{N}-\mathrm{Me}$ & $113.91 \pm 1.30$ & $122.16 \pm 2.02$ & $127.80 \pm 2.16$ & $115.25 \pm 1.07$ & $121.97 \pm 1.43$ & $124.88 \pm 1.07$ \\
\hline S-PNS & $41.07 \pm 0.73$ & $44.32 \pm 0.95$ & $45.83 \pm 0.99$ & $46.42 \pm 0.67$ & $48.81 \pm 0.68$ & $49.20 \pm 0.63$ \\
\hline S-Go & $68.78 \pm 1.00$ & $75.93 \pm 1.95$ & $78.09 \pm 1.39$ & $72.68 \pm 0.97$ & $78.50 \pm 1.11$ & $82.46 \pm 1.02$ \\
\hline
\end{tabular}

$\mathrm{X}$ : Mean, Sx: Standard error of the mean 
Table 3 Means and standard errors of the mean for PA cephalometric radiograph variables at each growth period in the UCCLP and control groups

\begin{tabular}{|c|c|c|c|c|c|c|}
\hline & \multicolumn{6}{|c|}{ Groups } \\
\hline & \multicolumn{3}{|c|}{ UCCLP } & \multicolumn{3}{|c|}{ Control } \\
\hline & $\mathrm{T} 1(n=24)$ & $\mathrm{T} 2(n=17)$ & $\mathrm{T} 3(n=18)$ & $\mathrm{T} 1(n=26)$ & $\mathrm{T} 2(n=26)$ & $\mathrm{T} 3(n=25)$ \\
\hline & $\mathrm{X} \pm \mathrm{Sx}$ & $\mathrm{X} \pm \mathrm{Sx}$ & $\mathrm{X} \pm \mathrm{Sx}$ & $\mathrm{X} \pm \mathrm{Sx}$ & $\mathrm{X} \pm \mathrm{Sx}$ & $\mathrm{X} \pm \mathrm{Sx}$ \\
\hline \multicolumn{7}{|l|}{ Facial widths } \\
\hline $\mathrm{Nc}^{\prime}-\mathrm{Nc}$ & $30.32 \pm 0.54$ & $31.22 \pm 0.90$ & $32.32 \pm 0.85$ & $26.86 \pm 0.57$ & $29.35 \pm 0.47$ & $30.32 \pm 0.67$ \\
\hline Zy'-Zy & $131.96 \pm 1.09$ & $135.73 \pm 1.70$ & $139.75 \pm 1.60$ & $124.66 \pm 1.22$ & $134.39 \pm 1.03$ & $138.04 \pm 1.17$ \\
\hline$M x^{\prime}-M x$ & $66.99 \pm 0.69$ & $67.04 \pm 1.33$ & $65.90 \pm 1.22$ & $64.01 \pm 0.95$ & $67.74 \pm 0.74$ & $69.07 \pm 0.89$ \\
\hline $\mathrm{Co}{ }^{\prime}-\mathrm{Co}$ & $120.12 \pm 0.93$ & $124.16 \pm 1.82$ & $125.80 \pm 1.69$ & $111.76 \pm 1.18$ & $121.08 \pm 1.16$ & $124.09 \pm 1.19$ \\
\hline$A g^{\prime}-A g$ & $86.61 \pm 0.88$ & $88.92 \pm 1.19$ & $91.87 \pm 1.29$ & $82.58 \pm 1.06$ & $88.72 \pm 0.93$ & $91.23 \pm 1.11$ \\
\hline \multicolumn{7}{|c|}{ Transverse variables } \\
\hline Nc'-VRD & $16.36 \pm 0.40$ & $17.80 \pm 0.55$ & $17.05 \pm 0.55$ & $13.34 \pm 0.41$ & $14.00 \pm 0.40$ & $14.95 \pm 0.52$ \\
\hline Nc-VRD & $13.89 \pm 0.39$ & $13.25 \pm 0.63$ & $15.18 \pm 0.70$ & $13.48 \pm 0.32$ & $15.32 \pm 0.29$ & $15.37 \pm 0.44$ \\
\hline Zy'-VRD & $67.49 \pm 0.83$ & $70.06 \pm 1.07$ & $69.67 \pm 0.77$ & $61.85 \pm 0.71$ & $66.16 \pm 0.68$ & $69.01 \pm 0.59$ \\
\hline Zy-VRD & $64.42 \pm 0.61$ & $65.65 \pm 0.95$ & $70.06 \pm 1.07$ & $62.78 \pm 0.69$ & $68.22 \pm 0.52$ & $69.01 \pm 0.79$ \\
\hline Mx'-VRD & $34.38 \pm 0.45$ & $35.89 \pm 0.95$ & $33.51 \pm 0.77$ & $31.95 \pm 0.59$ & $32.92 \pm 0.52$ & $34.16 \pm 0.51$ \\
\hline Mx-VRD & $32.58 \pm 0.60$ & $31.11 \pm 0.70$ & $32.33 \pm 0.83$ & $32.01 \pm 0.53$ & $34.79 \pm 0.41$ & $34.89 \pm 0.54$ \\
\hline Co'-VRD & $61.50 \pm 0.70$ & $64.33 \pm 1.10$ & $62.58 \pm 0.86$ & $55.32 \pm 0.75$ & $59.15 \pm 0.76$ & $62.30 \pm 0.71$ \\
\hline Co-VRD & $58.59 \pm 0.71$ & $59.79 \pm 1.12$ & $63.21 \pm 1.18$ & $56.42 \pm 0.64$ & $61.92 \pm 0.62$ & $61.74 \pm 0.74$ \\
\hline Ag'-VRD & $45.13 \pm 0.67$ & $47.92 \pm 0.86$ & $46.31 \pm 0.70$ & $41.68 \pm 0.77$ & $43.05 \pm 0.74$ & $45.17 \pm 0.69$ \\
\hline Ag-VRD & $41.46 \pm 0.70$ & $40.98 \pm 0.81$ & $45.51 \pm 1.09$ & $40.87 \pm 0.75$ & $45.65 \pm 0.58$ & $46.04 \pm 0.74$ \\
\hline \multicolumn{7}{|c|}{ Vertical variables } \\
\hline Nc'-HRD & $42.89 \pm 0.58$ & $46.52 \pm 0.75$ & $48.06 \pm 1.04$ & $44.69 \pm 0.64$ & $47.78 \pm 0.68$ & $50.66 \pm 0.54$ \\
\hline Nc-HRD & $42.54 \pm 0.41$ & $44.19 \pm 0.51$ & $47.52 \pm 1.04$ & $45.06 \pm 0.65$ & $48.14 \pm 0.65$ & $50.85 \pm 0.56$ \\
\hline Zy'-HRD & $29.06 \pm 0.75$ & $32.59 \pm 0.81$ & $32.54 \pm 0.69$ & $28.63 \pm 1.06$ & $31.07 \pm 1.10$ & $33.09 \pm 1.18$ \\
\hline Zy-HRD & $30.47 \pm 0.67$ & $33.37 \pm 0.84$ & $33.05 \pm 0.70$ & $29.82 \pm 1.05$ & $31.53 \pm 1.13$ & $33.47 \pm 1.16$ \\
\hline Mx'-HRD & $50.30 \pm 1.02$ & $54.46 \pm 0.74$ & $57.27 \pm 1.23$ & $52.82 \pm 0.75$ & $56.62 \pm 0.90$ & $58.94 \pm 0.71$ \\
\hline Mx-HRD & $51.06 \pm 0.94$ & $55.32 \pm 0.90$ & $58.13 \pm 1.25$ & $53.85 \pm 0.87$ & $56.73 \pm 0.80$ & $59.19 \pm 0.71$ \\
\hline Co'-HRD & $32.33 \pm 0.98$ & $36.26 \pm 1.07$ & $35.38 \pm 1.07$ & $31.13 \pm 1.29$ & $34.36 \pm 1.20$ & $38.38 \pm 0.94$ \\
\hline Co-HRD & $33.47 \pm 0.91$ & $36.32 \pm 1.05$ & $36.42 \pm 1.22$ & $31.96 \pm 1.24$ & $35.29 \pm 1.13$ & $39.45 \pm 1.13$ \\
\hline Ag'-HRD & $88.36 \pm 1.02$ & $96.55 \pm 1.40$ & $101.45 \pm 1.69$ & $89.38 \pm 1.15$ & $98.31 \pm 1.17$ & $105.53 \pm 0.98$ \\
\hline Ag-HRD & $89.24 \pm 0.97$ & $97.03 \pm 1.46$ & $100.94 \pm 1.66$ & $90.01 \pm 1.04$ & $97.48 \pm 1.15$ & $105.81 \pm 0.90$ \\
\hline \multicolumn{7}{|c|}{ Mandibular morphologic variables } \\
\hline $\mathrm{Ag}-\mathrm{Me}$ & $48.56 \pm 0.76$ & $50.33 \pm 1.12$ & $52.27 \pm 0.86$ & $46.61 \pm 0.86$ & $49.14 \pm 0.72$ & $49.21 \pm 0.77$ \\
\hline $\mathrm{Ag}-\mathrm{Me}$ & $49.91 \pm 0.93$ & $50.34 \pm 1.06$ & $54.34 \pm 1.12$ & $47.28 \pm 0.84$ & $50.30 \pm 0.71$ & $49.41 \pm 0.85$ \\
\hline Co'-Ag' & $58.43 \pm 0.78$ & $62.56 \pm 1.54$ & $68.10 \pm 1.60$ & $59.89 \pm 0.97$ & $66.02 \pm 0.81$ & $69.37 \pm 0.70$ \\
\hline Co-Ag & $58.41 \pm 0.92$ & $63.60 \pm 1.67$ & $66.96 \pm 1.64$ & $60.14 \pm 1.05$ & $64.36 \pm 1.00$ & $68.25 \pm 0.83$ \\
\hline $\mathrm{Co}^{\prime}-\mathrm{Me}$ & $98.88 \pm 1.18$ & $103.57 \pm 2.21$ & $111.03 \pm 1.98$ & $97.27 \pm 1.47$ & $104.73 \pm 1.28$ & $106.13 \pm 1.26$ \\
\hline $\mathrm{Co}-\mathrm{Me}$ & $99.66 \pm 1.15$ & $105.01 \pm 2.29$ & $112.13 \pm 2.17$ & $98.29 \pm 1.46$ & $104.54 \pm 1.48$ & $104.65 \pm 1.35$ \\
\hline Co'.Ag'.Me & $135.19 \pm 1.17$ & $132.87 \pm 1.09$ & $134.22 \pm 1.14$ & $131.56 \pm 0.99$ & $130.34 \pm 0.83$ & $126.25 \pm 0.95$ \\
\hline Co.Ag.Me & $133.87 \pm 1.08$ & $134.09 \pm 1.42$ & $135.06 \pm 1.12$ & $132.06 \pm 0.94$ & $131.20 \pm 1.02$ & $124.87 \pm 0.88$ \\
\hline
\end{tabular}

X: Mean, Sx: Standard error of the mean

lateral cephalometric radiographs. First and second measurements were compared, and correlation coefficients $\left(\mathrm{r}^{2}\right)$ were calculated.

\section{Statistical analysis}

The factorial analysis of variance technique was used to compare intragroup and intergroup cephalometric measurements at each growth period, and the Duncan method was used when interaction was observed. The $t$-test was used for comparison of asymmetry between two groups, and the paired $t$-test was used to determine the degree of asymmetry in the UCCLP and control groups.

\section{Results}

The reliability of the method was high; the correlation coefficients were $0.9916-0.9985$. The means and standard errors of the mean for variables in lateral cephalometric and PA cephalometric radiographs, at each time interval, are presented in Tables 2 and 3, respectively.

Values in intragroup and intergroup comparisons of variables on lateral cephalometric radiographs are shown 
Table 4 Comparison of lateral cephalometric variables, by group and growth period

\begin{tabular}{|c|c|c|c|c|c|}
\hline & Group & Time & $\mathrm{T} 1-\mathrm{T} 2$ & T1-T3 & $\mathrm{T} 2-\mathrm{T} 3$ \\
\hline \multicolumn{6}{|c|}{ Cranial variables } \\
\hline $\mathrm{N}-\mathrm{S}$ & 0.290 & 0.000 & 0.000 & 0.000 & 0.000 \\
\hline S-Ba & 0.026 & 0.001 & 0.001 & 0.001 & NS \\
\hline $\mathrm{N}-\mathrm{Ba}$ & 0.026 & 0.000 & 0.000 & 0.000 & 0.000 \\
\hline NSBa & 0.619 & 0.649 & NS & NS & NS \\
\hline \multicolumn{6}{|c|}{ Maxillary variables } \\
\hline Co-A & 0.000 & 0.000 & 0.000 & 0.000 & NS \\
\hline A-VR & 0.000 & 0.71 & NS & NS & NS \\
\hline A-HR & 0.04 & 0.000 & 0.000 & 0.000 & NS \\
\hline SNA & 0.000 & 0.404 & NS & NS & NS \\
\hline SN.NL & 0.000 & 0.645 & NS & NS & NS \\
\hline \multicolumn{6}{|c|}{ Mandibular variables } \\
\hline Go-Gn & 0.000 & 0.000 & 0.000 & 0.000 & 0.000 \\
\hline Co-Gn & 0.001 & 0.000 & 0.000 & 0.000 & 0.000 \\
\hline B-VR & 0.000 & 0.001 & NS & 0.001 & 0.001 \\
\hline Pg-VR & 0.000 & 0.000 & 0.000 & 0.000 & 0.000 \\
\hline Co-Go & 0.000 & 0.000 & 0.000 & 0.000 & 0.000 \\
\hline Go-HR & 0.001 & 0.000 & 0.000 & 0.000 & 0.000 \\
\hline Co-HR & 0.821 & 0.024 & 0.024 & 0.024 & NS \\
\hline B-HR & 0.539 & 0.000 & 0.000 & 0.000 & 0.000 \\
\hline Pg-HR & 0.746 & 0.000 & 0.000 & 0.000 & 0.000 \\
\hline SNB & 0.000 & 0.064 & NS & NS & NS \\
\hline SN.ML & 0.000 & 0.076 & NS & NS & NS \\
\hline CoGo.GoMe & 0.000 & 0.006 & NS & 0.006 & 0.006 \\
\hline \multicolumn{6}{|c|}{ Maxillomandibular variables } \\
\hline ANB & 0.007 & 0.000 & 0.000 & 0.000 & NS \\
\hline NL.ML & 0.000 & 0.006 & NS & 0.006 & 0.006 \\
\hline \multicolumn{6}{|c|}{ Facial height variables } \\
\hline N-ANS & 0.013 & 0.000 & 0.000 & 0.000 & 0.000 \\
\hline ANS-Me & 0.025 & 0.000 & 0.000 & 0.000 & 0.000 \\
\hline $\mathrm{N}-\mathrm{Me}$ & 0.624 & 0.000 & 0.000 & 0.000 & 0.000 \\
\hline S-PNS & 0.000 & 0.000 & 0.000 & 0.000 & NS \\
\hline S-Go & 0.000 & 0.000 & 0.000 & 0.000 & 0.000 \\
\hline
\end{tabular}

NS: not significant

on Table 4. The posterior and total cranial base (S-Ba, $\mathrm{N}-\mathrm{Ba}$ ), upper anterior (N-ANS) and posterior facial height (S-PNS, S-Go), maxillary variables (Co-A, A-VR, A-HR, SNA), and most mandibular variables (Go-Gn, Co-Gn, B-VR, Pg-VR, Co-Go, Go-HR, SNB) were significantly lower in the UCCLP group at each growth period. The maxilla and mandible were significantly posteriorly rotated in the UCCLP group.

A comparison between groups of facial-width variables on PA cephalometric radiographs, by growth period, is shown in Table 5. Total nasal width (Nc'$\mathrm{Nc})$ was significantly higher in the UCCLP group $(P<$ 0.001), while antegonial (Ag'-Ag), zygomatic (Zy'-Zy), maxillary (Mx'-Mx) and condylar (Co'-Co) widths did not differ significantly between the control and UCCLP groups. Table 6 shows interactions among the variables described in Table 5.
Table 5 Facial-width variables on PA cephalometric radiographs by group and growth period

\begin{tabular}{lccccc}
\hline & Group & Time & T1-T2 & T1-T3 & T2-T3 \\
\hline Nc'-Nc & $* * *$ & $* * *$ & $* * *$ & $* * *$ & NS \\
Zy'-Zy & interaction & & & \\
Mx'-Mx & interaction & & & \\
Co'-Co & interaction & & & \\
Ag'-Ag & NS & $* * *$ & $* * *$ & $* * *$ & $* * *$ \\
$* * * P<0.001$, NS: not significant & & &
\end{tabular}

Table 6 Interactions between variables

\begin{tabular}{llccc}
\hline & Groups & T1-T2 & T1-T3 & T2-T3 \\
\hline Zy'-Zy & UCCLP & & 0.032 & \\
& Control & 0.032 & 0.032 & 0.032 \\
Mx'-Mx & UCCLP & & & \\
& Control & 0.062 & 0.062 & \\
Co'-Co & UCCLP & 0.028 & 0.028 & \\
& Control & & 0.028 & \\
\hline
\end{tabular}

An interaction in the relationship among $\geq 3$ variables is present when the simultaneous influence of two variables on a third is not additive.

Asymmetry values were calculated as the mean difference between measurements on the left and right sides and were obtained by using reference planes on PA cephalometric radiographs. Asymmetry in the UCCLP patients was calculated by subtracting non-cleft-side from cleftside values. Horizontal asymmetries were calculated from variables measured by using the vertical reference plane on PA cephalometric radiographs. Vertical asymmetries were calculated by means of variables measured by using the horizontal reference plane. Table 7 shows the means and standard errors of the mean for asymmetry variables and the comparison of asymmetry variables between the UCCLP and control groups at each growth period.

Horizontal asymmetries (NcHA, ZyHA, MxHA, CoHA, AgHA) were significant in the UCCLP group at the T1 and T2 growth periods. Vertical nasal asymmetry (NcVA) was significant at T2, vertical zygomatic (ZyVA) and antegonial asymmetry (AgVA) were significant at $\mathrm{T} 1$, and vertical condylar asymmetry (CoVA) was significant at T1 and T3. Except for MMA1 at T3, no significant mandibular asymmetries were detected in the UCCLP group. However, mandibular asymmetries were more significant in the Class I group.

\section{Discussion}

Although craniofacial morphology has been extensively investigated in CLP patients, our study is valuable 
Table 7 Means and standard errors of the mean of asymmetry variables and a comparison of asymmetry variables between the UCCLP and control groups at each growth period

\begin{tabular}{|c|c|c|c|c|c|c|}
\hline \multirow{2}{*}{ Variables } & \multirow{2}{*}{ Time } & \multicolumn{2}{|c|}{ UCCLP } & \multicolumn{2}{|c|}{ Control } & \multirow{2}{*}{$P$} \\
\hline & & $n$ & $\mathrm{X} \pm \mathrm{Sx}$ & $\mathrm{n}$ & $\mathrm{X} \pm \mathrm{Sx}$ & \\
\hline \multicolumn{7}{|c|}{ Horizontal asymmetry } \\
\hline \multirow{3}{*}{$(\mathrm{NcHA})(\mathrm{mm})$} & $\mathrm{T} 1$ & 24 & $2.47 \pm 0.57 * * *$ & 26 & $-0.14 \pm 0.47$ & $* * *$ \\
\hline & $\mathrm{T} 2$ & 17 & $4.55 \pm 0.77 * * *$ & 26 & $-1.32 \pm 0.51^{*}$ & $* * *$ \\
\hline & $\mathrm{T} 3$ & 18 & $1.87 \pm 0.93$ & 25 & $-0.42 \pm 0.69$ & $*$ \\
\hline \multirow[t]{3}{*}{$(\mathrm{ZyHA})(\mathrm{mm})$} & $\mathrm{T} 1$ & 24 & $3.07 \pm 0.97 * *$ & 26 & $-0.93 \pm 0.70$ & $* * *$ \\
\hline & $\mathrm{T} 2$ & 17 & $4.40 \pm 1.10^{* * *}$ & 26 & $-2.06 \pm 0.63 * *$ & $* * *$ \\
\hline & $\mathrm{T} 3$ & 18 & $-0.39 \pm 0.94$ & 25 & $0.00 \pm 0.76$ & NS \\
\hline \multirow[t]{3}{*}{ (MxHA) (mm) } & $\mathrm{T} 1$ & 24 & $1.80 \pm 0.81^{*}$ & 26 & $-0.06 \pm 0.59$ & NS \\
\hline & $\mathrm{T} 2$ & 17 & $4.77 \pm 1.00^{* * *}$ & 26 & $-1.87 \pm 0.59^{* *}$ & $* * *$ \\
\hline & $\mathrm{T} 3$ & 18 & $1.17 \pm 1.04$ & 25 & $-0.73 \pm 0.56$ & NS \\
\hline \multirow[t]{3}{*}{$(\mathrm{CoHA})(\mathrm{mm})$} & $\mathrm{T} 1$ & 24 & $2.91 \pm 1.06^{*}$ & 26 & $-1.10 \pm 0.75$ & $* *$ \\
\hline & $\mathrm{T} 2$ & 17 & $4.54 \pm 1.27 * *$ & 26 & $-2.77 \pm 0.77 * * *$ & $* * *$ \\
\hline & $\mathrm{T} 3$ & 18 & $-0.63 \pm 1.18$ & 25 & $0.55 \pm 0.83$ & NS \\
\hline \multirow[t]{3}{*}{$(\mathrm{AgHA})(\mathrm{mm})$} & $\mathrm{T} 1$ & 24 & $3.67 \pm 1.06^{* *}$ & 26 & $0.80 \pm 1.11$ & NS \\
\hline & $\mathrm{T} 2$ & 17 & $6.95 \pm 1.17^{* * *}$ & 26 & $-2.60 \pm 0.96^{*}$ & $* * *$ \\
\hline & $\mathrm{T} 3$ & 18 & $0.79 \pm 1.30$ & 25 & $-0.87 \pm 0.90$ & NS \\
\hline \multicolumn{7}{|l|}{ Vertical asymmetry } \\
\hline \multirow[t]{3}{*}{$(\mathrm{NcVA})(\mathrm{mm})$} & $\mathrm{T} 1$ & 24 & $0.35 \pm 0.41$ & 26 & $-0.37 \pm 0.27$ & NS \\
\hline & $\mathrm{T} 2$ & 17 & $2.32 \pm 0.58^{* * *}$ & 26 & $-0.36 \pm 0.27$ & $* * *$ \\
\hline & $\mathrm{T} 3$ & 18 & $0.54 \pm 0.53$ & 25 & $-0.19 \pm 0.17$ & NS \\
\hline \multirow[t]{3}{*}{$(\mathrm{ZyVA})(\mathrm{mm})$} & $\mathrm{T} 1$ & 24 & $-1.41 \pm 0.65^{*}$ & 26 & $-1.20 \pm 0.47^{*}$ & NS \\
\hline & $\mathrm{T} 2$ & 17 & $-0.78 \pm 0.55$ & 26 & $-0.45 \pm 0.42$ & NS \\
\hline & $\mathrm{T} 3$ & 18 & $-0.52 \pm 0.41$ & 25 & $-0.39 \pm 0.45$ & NS \\
\hline \multirow[t]{3}{*}{$(\mathrm{MxVA})(\mathrm{mm})$} & $\mathrm{T} 1$ & 24 & $-0.76 \pm 0.38$ & 26 & $-1.03 \pm 0.46^{*}$ & NS \\
\hline & $\mathrm{T} 2$ & 17 & $-0.86 \pm 0.51$ & 26 & $-0.11 \pm 0.37$ & NS \\
\hline & $\mathrm{T} 3$ & 18 & $-0.86 \pm 0.62$ & 25 & $-0.26 \pm 0.39$ & NS \\
\hline \multirow[t]{3}{*}{$(\mathrm{CoVA})(\mathrm{mm})$} & $\mathrm{T} 1$ & 24 & $-1.14 \pm 0.45^{*}$ & 26 & $-0.83 \pm 0.41$ & NS \\
\hline & $\mathrm{T} 2$ & 17 & $-0.06 \pm 0.76$ & 26 & $-0.92 \pm 0.34^{*}$ & NS \\
\hline & $\mathrm{T} 3$ & 18 & $-1.04 \pm 0.46^{*}$ & 25 & $-1.07 \pm 0.71$ & NS \\
\hline \multirow[t]{3}{*}{$(\mathrm{AgVA})(\mathrm{mm})$} & $\mathrm{T} 1$ & 24 & $-0.88 \pm 0.38^{*}$ & 26 & $-0.63 \pm 0.45$ & NS \\
\hline & $\mathrm{T} 2$ & 17 & $-0.47 \pm 0.46$ & 26 & $0.83 \pm 0.34 *$ & $*$ \\
\hline & $\mathrm{T} 3$ & 18 & $-0.84 \pm 0.71$ & 25 & $1.37 \pm 0.54^{*}$ & $*$ \\
\hline \multicolumn{7}{|c|}{ Mandibular asymmetry } \\
\hline \multirow[t]{3}{*}{ MMA1 } & $\mathrm{T} 1$ & 24 & $-1,35 \pm 0,96$ & 26 & $-0,67 \pm 0,45$ & NS \\
\hline & $\mathrm{T} 2$ & 17 & $-0,02 \pm 0,75$ & 26 & $-1,15 \pm 0,54^{*}$ & NS \\
\hline & $\mathrm{T} 3$ & 18 & $-2,07 \pm 0,96^{*}$ & 25 & $-0,20 \pm 0,53$ & NS \\
\hline \multirow[t]{3}{*}{ MMA2 } & $\mathrm{T} 1$ & 24 & $0,03 \pm 0,55$ & 26 & $-0,26 \pm 0,48$ & NS \\
\hline & $\mathrm{T} 2$ & 17 & $-1,04 \pm 0,73$ & 26 & $1,66 \pm 0,44 * * *$ & $* *$ \\
\hline & $\mathrm{T} 3$ & 18 & $1,14 \pm 1,04$ & 25 & $1,12 \pm 0,64$ & NS \\
\hline \multirow[t]{3}{*}{ MMA3 } & $\mathrm{T} 1$ & 24 & $-0,78 \pm 0,76$ & 26 & $-1,02 \pm 0,42 *$ & NS \\
\hline & $\mathrm{T} 2$ & 17 & $-1,44 \pm 0,69$ & 26 & $0,19 \pm 0,50$ & NS \\
\hline & $\mathrm{T} 3$ & 18 & $-1,10 \pm 1,00$ & 25 & $1,48 \pm 0,69^{*}$ & $*$ \\
\hline \multirow[t]{3}{*}{ MMA4 } & $\mathrm{T} 1$ & 24 & $1,32 \pm 0,87$ & 26 & $-0,50 \pm 0,67$ & NS \\
\hline & $\mathrm{T} 2$ & 17 & $-1,22 \pm 0,78$ & 26 & $-0,86 \pm 0,56$ & NS \\
\hline & $\mathrm{T} 3$ & 18 & $-0,84 \pm 0,71$ & 25 & $1,37 \pm 0,54^{*}$ & $*$ \\
\hline
\end{tabular}

$* P<0.05,{ }^{*} P<0.01, * * * P<0.001$, NS: not significant. MMA1: the differences in the lengths between Ag'-Me and Ag-Me; MMA2: the differences in the lengths between Co'-Ag' and Co-Ag; MMA3: the differences in the lengths between Co'-Me and Co-Me; MMA4: the differences in the angles between Co'.Ag'.Me and Co.Ag.Me.

because we included three age-matched subgroups in the comparison of CLP and Class I subjects. Previous studies found no relationship between craniofacial changes and gender in CLP patients $(12,13)$. We were unable to subdivide the groups by gender; however, the numbers of males and females in each group were similar in our analysis.

The abnormal facial growth pattern in CLP patients may result from intrinsic tissue defects and/or negative environmental factors. Intrinsic tissue anomalies can 
be interpreted as abnormal growth factors affecting the midface and even the basicranium. Some investigators noted anomalies in cranial base dimensions $(14,15)$, while others found no abnormal growth in cranial base structures $(14,16)$. The present UCCLP patients had a shorter posterior and total cranial base at all growth periods, but the anterior cranial base and cranial base angle did not differ significantly.

In UCCLP patients the maxillary dimensions were significantly shorter and the maxilla had significant posterior rotation at all growth periods. These results support the findings of previous studies $(17,18)$. The deficiency in maxillary growth might be due to palatoplasty (19) and scar tissue on the lips $(20,21)$. The other reason for retrognathic maxilla could be inappropriate development of the cranial base. In our clinic, maxillary growth is stimulated with extraoral appliances before and during puberty. No patient in our CLP group with a maxillary growth deficiency needed orthognathic surgery after orthopedic therapy.

The significant decrease in posterior face height (S-PNS) in the UCCLP group might be due to the more posterior inclination of the nasal plane in relation to the cranial base. This result is also supported in the literature $(2,22,23)$.

Some studies found that surgery for CLP does not affect mandibular growth $(21,24)$. Our results showed that the mandibula was significantly shorter, more retrusive, and rotated posteriorly in the UCCLP group at all growth periods. Because of this rotation, anteroinferior face height was significantly greater. The gonial angle was significantly larger in the UCCLP group, which supports the findings of earlier investigations $(2,23)$. We hypothesize that the shorter mandible is caused by the shorter cranial base and mandibular adaptation to underdeveloped maxilla.

Nakamura (25) and Jain and Krogman (26) described the facial width of CLP patients as wide. However, we found no significant difference in total zygomatic, maxillary, condylar, or antegonial widths between the UCCLP and control groups. As expected, total nasal width was significantly larger in the UCCLP group at all three growth periods.

In the evaluation of horizontal asymmetries, nasal, zygomatic, maxillary, condylar, and antegonial widths were significantly greater on the cleft side; however, these horizontal asymmetries were not observed in the postpubertal UCCLP group. This result is likely due to adaptation to asymmetry after pubertal growth. Our other important result is the large extent of horizontal asymmetries during puberty in both groups. Growth is rapid during puberty; thus, asymmetries are more easily detected during this period. However, because of compensation mechanisms the statistical significance of asymmetries decreases after puberty.

A three-dimensional study of adults revealed no distinctive midfacial hard- or soft-tissue asymmetries between the affected and unaffected sides in individuals with nonsyndromic unilateral cleft lip and palate (7). However, some researchers found greater asymmetry in CLP patients than in controls, particularly in the midface (27).

The most significant vertical asymmetry was observed in the nasal area during puberty. Overall, vertical asymmetries were less severe than horizontal asymmetries. Our evaluation of mandibular asymmetry indicated that the mandible was not affected in UCCLP, as more significant asymmetries were detected in the Class I control group. These results accord with previous findings $(7,28)$.

A limitation of this study is the small numbers of subjects in the subgroups. Further investigation, with larger databases and an age- and sex-matched control group, is needed for craniofacial evaluation before, during, and after puberty.

Many aspects of craniofacial growth differed significantly on the sagittal, vertical, and transverse planes in UCCLP patients. To ensure successful treatment, lateral and PA cephalometric radiographs should be used to evaluate CLP patients at earlier developmental stages.

\section{References}

1. Li Y, Shi B, Song QG, Zuo H, Zheng Q (2006) Effects of lip repair on maxillary growth and facial soft tissue development in patients with a complete unilateral cleft of lip, alveolus and palate. J Craniomaxillofac Surg 34, 355-361.

2. Öztürk Y, Cura N (1996) Examination of craniofacial morphology in children with unilateral cleft lip and palate. Cleft Palate Craniofac J 33, 32-36.

3. Mølsted K, Dahl E (1990) Asymmetry of the maxilla in children with complete unilateral cleft lip and palate. Cleft Palate J 27, 184-190.

4. Ras F, Habets LL, van Ginkel FC, Prahl-Andersen B (1994) Three-dimensional evaluation of facial asymmetry in cleft lip and palate. Cleft Palate Craniofac J 31, 116-121.

5. Son WS, Kim MK (1995) Facial asymmetry of unilateral cleft lip and palate patients. Korean J Orthod 25, 13-18.

6. Broder HL, Smith FB, Strauss RP (1994) Effects of visible and invisible orofacial defects on self-perception and adjustment across developmental eras and gender. Cleft Palate Craniofac J 31, 429-436.

7. Choi YK, Park SB, Kim YI, Son WS (2013) Three-dimensional evaluation of midfacial asymmetry in patients with nonsyndromic unilateral cleft lip and palate by cone-beam 
computed tomography. Korean J Orthod 43, 113-119.

8. Horswell BB, Levant BA (1988) Craniofacial growth in unilateral cleft lip and palate: skeletal growth from eight to eighteen years. Cleft Palate J 25, 114-121.

9. Enemark H, Boluns S, Jørgensen I (1990) Evaluation of unilateral cleft lip and palate treatment: long term results. Cleft Palate J 27, 354-361.

10. Schneiderman ED, Xu H, Salyer KE (2009) Characterization of the maxillary complex in unilateral cleft lip and palate using cone-beam computed tomography: a preliminary study. J Craniofac Surg 20, Suppl 2, 1699-1710.

11. Agarwal R, Parihar A, Mandhani PA, Chandra R (2012) Three-dimensional computed tomographic analysis of the maxilla in unilateral cleft lip and palate: implications for rhinoplasty. J Craniofac Surg 23, 1338-1342.

12. Krogman WM, Jain RB, Long RE Jr (1982) Sex differences in craniofacial growth from one month to ten years of cleft lip and palate. Cleft Palate J 19, 62-71.

13. Long RE Jr, Jain RB, Krogman WM (1982) Possible sexdiscriminant variables in craniofacial growth in clefting. Am J Orthod 82, 392-402.

14. Sandham A, Cheng L (1988) Cranial base and cleft lip and palate. Angle Orthod 58, 163-168.

15. Liu R, Lu D, Wamalwa P, Li C, Hu H, Zou S (2011) Craniofacial morphology characteristics of operated unilateral complete cleft lip and palate patients in mixed dentition. Oral Surg Oral Med Oral Pathol Oral Radiol Endod 112, e16-25.

16. Goyenc YB, Gurel HG, Memili B (2008) Craniofacial morphology in children with operated complete unilateral cleft lip and palate. J Craniofac Surg 19, 1396-1401.

17. Hayashi I, Sakuda M, Takimoto K, Miyazaki T (1976) Craniofacial growth in complete unilateral cleft lip and palate: a roengeno-cephalometric study. Cleft Palate J 13, 215-237.

18. Treutlein C, Berten JL, Swennen G, Brachvogel P (2003) Comparative cephalometric study of 10 -year-old patients with complete unilateral cleft lip and palate. A cross-sectional study of the Hanover concept. J Orofac Orthop 64, 27-39.

19. Mars M, Houston WJ (1990) A preliminary study of facial growth and morphology in unoperated male unilateral cleft lip and palate subjects over 13 years of age. Cleft Palate J 27, 7-10.

20. Bardach J (1990) The influence of cleft lip repair on facial growth. Cleft Palate J 27, 76-78.

21. Capelozza Filho L, Normando AD, da Silva Filho OG (1996) Isolated influences of lip and palate surgery on facial growth: comparison of operated and unoperated male adults with UCLP. Cleft Palate Craniofac J 33, 51-56.

22. Hammond AB, Smahel Z, Moss ML (1993) Finite element method analysis of craniofacial morphology in unilateral cleft lip and palate prior to palatoplasty. J Craniofac Genet Dev Biol 13, 47-56.

23. Swennen G, Berten JL, Schliephake H, Treutlein C, Dempf R, Malevez C et al. (2002) Midfacial morphology in children with unilateral cleft lip and palate treated by different surgical protocols. Int J Oral Maxillofac Surg 31, 13-22.

24. da Silva OG Jr, Normando AD, Capelozza L Jr (1992) Mandibular morphology and spatial position in patients with clefts: intrinsic or iatrogenic? Cleft Palate Craniofac J 29, 369-375.

25. Nakamura S, Savara BS, Thomas DR (1972) Facial growth of children with cleft lip and-or palate. Cleft Palate J 9, 119-131.

26. Jain RB, Krogman WM (1983) Craniofacial growth in clefting from one month to ten years as studied by P-A headfilms. Cleft Palate J 20, 314-326.

27. Meyer-Marcotty P, Alpers GW, Gerdes AB, StellzigEisenhauer A (2010) Impact of facial asymmetry in visual perception: a 3-dimensional data analysis. Am J Orthod Dentofacial Orthop 137, e1-8.

28. Kurt G, Bayram M, Uysal T, Ozer M (2010) Mandibular asymmetry in cleft lip and palate patients. Eur J Orthod 32, 19-23. 